\section{Development and 4D visualization of pressure-solution rock fabrics at lab- compatible timescale using a calcite structural analogue: understanding the role of the microstructure and grain contacts}

\section{MARCO VOLTOLINI ${ }^{1}$ AND BENJAMIN GILBERT ${ }^{2}$}

${ }^{1}$ Lawrence Berkeley National Laboratory

${ }^{2}$ Lawrence Berkeley National Lab

Presenting Author: mvoltolini@lbl.gov

Pressure-Solution (PS) is a complex mechanism playing a key role during compaction and diagenesis of rocks and in the generation of specific fabrics. One of the main hurdles in studying these processes is that they take a very long time to develop, therefore laboratory experiments become limited, while natural samples only provide one single frame of a very long movie. The evolution of PS-related fabrics depend on the behavior of the single grain contacts: ideally when a pack of grains in chemical equilibrium with a fluid is subject to stress, we expect to observe enhanced local dissolution at the grain contacts and reprecipitation of new material. When considering the mineral calcite, the time required to generate substantial PS is not compatible with laboratory experiments. To overcome this problem, we used the structural analogue nitratine: the mineral is isostructural with calcite, it shares the same crystal morphology, cleavage, mechanical twinning direction... but crystallization and dissolution kinetics are much faster, enabling dynamic experiments compatible with the lab timescale. The goal of the first series of experiments was to understand the role of morphological characteristics of the starting material in developing specific PS fabrics, and to understand the processes at the single grain contacts. We used in-situ synchrotron X-ray microtomography to image time-lapse sequences of nitratine crystals packs to observe the evolution of the system while generating of a synthetic rock. An example of a single frame (after $\sim 6 \mathrm{~h}$ of compaction) is presented in Fig. 1, along with additional details. At this stage, the single grains are still wellrecognizable, as well as their contacts, often showing a typical suture-type interface. After weeks of reaction the rock becomes more compact and starts to develop foliation, where phyllosilicates are present. The full sequences available allow measuring the morphometric characteristics of the sample both in a dynamic and local fashion: we can e.g. calculate how the grain size, porosity, or contacts change during the generation of the fabric, or how phyllosilicates develop preferred orientation. Correlation analysis also allowed us to quantitatively evaluate and visualize the parts of the sample where more compaction occurred.

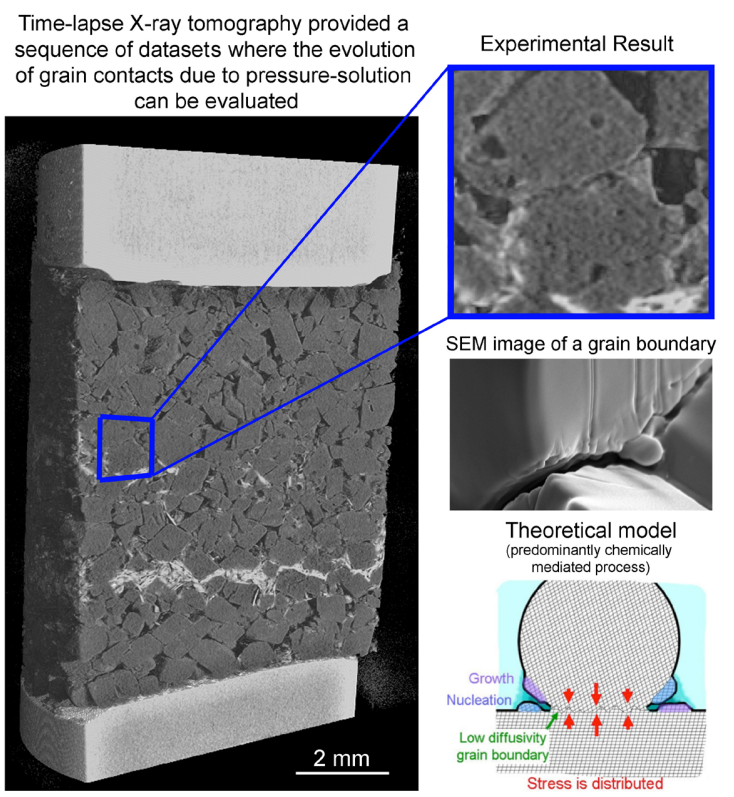

